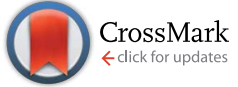

Cite this: RSC Adv., 2017, 7, 8421

Received 20th December 2016 Accepted 19th January 2017

DOI: $10.1039 / c 6 r a 28435 c$

www.rsc.org/advances

\section{Diffusion of tellurium at nickel grain boundaries: a first-principles study}

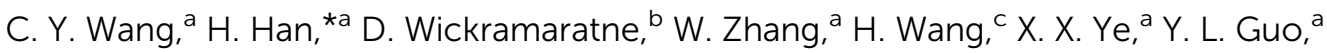 \\ K. Shao and P. Huai ${ }^{\star a}$
}

The knowledge of the behavior of Te in nickel grain boundaries (GB) is of significant importance for the application of nickel alloys in molten salt reactors. The atomic structures, stabilities, segregation behaviors and diffusion barriers of Te are studied for the bulk, surfaces and four kinds of GBs of nickel. Our first-principles calculations indicate the segregation of Te is most favorable at $\Sigma=5$ (021) GB and

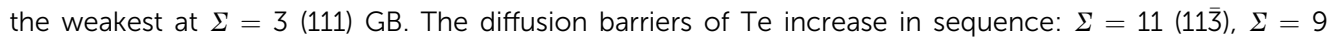
(221), $\Sigma=3$ (111) and $\Sigma=5$ (021). The calculated diffusion barrier of Te on $\Sigma=11$ (11) is $0.35 \mathrm{eV}$ lower than in the bulk, indicating a fast diffusion of Te along this GB. We also consider the effect of strain on the diffusion and find it to be sensitive to the different GB types. When the tensile strain is up to $4 \%$, the diffusion barriers of Te are lowered by $0.51 \mathrm{eV}$ and $0.15 \mathrm{eV}$ for $\Sigma=5$ (021) and $\Sigma=11$ (11) $)$, respectively. In contrast, this effect for $\Sigma=3$ (111) is negligible.

\section{Introduction}

The growing demands for economical, safe and reliable energy resources have accelerated the development of a new generation of nuclear reactors. The Molten Salt Reactor (MSR), which is one of the most promising new generation reactors, uses molten fluoride salts as coolant and dissolves the actinide fission fuel in the coolant. The strong corrosion of the high temperature liquid fuel brings challenges to the structural materials in MSRs. Historically, Molten Salt Reactor Experiments (MSRE) at Oak Ridge National Laboratory (ORNL, USA) have used Hastelloy $\mathrm{N}$ (a nickel-based alloy) as the structural material of choice. However, Hastelloy $\mathrm{N}$ exhibits apparent intergranular cracking after exposure to fuel salt. There have been many experimental and theoretical studies ${ }^{1-4}$ focused on the issue which often claim that the fission-product tellurium (Te) can cause the embrittlement of Hastelloy N. Studies from ORNL also prove that Te is an embrittler for the nickel alloy and it is the inward diffusion of Te along the nickel grain boundaries (GBs) that leads to the embrittlement of the nickel alloy. ${ }^{5}$ In addition to embrittlement of $\mathrm{Ni}$ being caused by $\mathrm{Te}$, nickel alloy embrittlement is also influenced by the external strain and stress given their impact on GB diffusion. Therefore, a deeper knowledge of these properties of Te in GBs is crucial for the understanding of embrittlement of the nickel alloy.

\footnotetext{
${ }^{a}$ Shanghai Institute of Applied Physics, Chinese Academy of Sciences, Shanghai 201800, P. R. China.E-mail: hanhanfudan@gmail.com; huaiping@sinap.ac.cn ${ }^{b}$ Materials Department, University of California, Santa Barbara, CA 93106, USA ${ }^{c}$ School of Physics and Engineering, Henan University of Science and Technology, Luoyang 471003, P. R. China
}

GBs are 2-dimentional defects in the crystal structure which are composed by two differently oriented grains. In solids, GBs belong to the most important defects and the properties of GBs are different from that in the crystal interior. In theory there can be a wide range of GB orientations that can occur. For the purposes of this study, we limit our investigations to GB orientations that are most commonly observed in Ni alloys which are computationally tractable, specifically, the symmetrical tilt grain boundary (STGB). ${ }^{6}$ In the STGBs, $\Sigma=3$ (111), $\Sigma=5$ (021), $\Sigma=9$ (221) and $\Sigma=11(11 \overline{3})$ GBs are all the experimentally confirmed STGB structures in nickel. $\Sigma=3$ (111) GB is the most commonly observed by experiments. ${ }^{7} \Sigma=5$ (021) is the most frequently used theoretical STGB model which has been utilized in many works. ${ }^{2,8,9}$ GB diffusion describes the movement of atoms along the GBs under a driving force, i.e., the difference in chemical potentials. It is well known that atoms move in GBs predominantly by simple exchanges with vacancies, i.e., vacancymediated diffusion. ${ }^{10}$ The fact that the impurity atom preferentially diffuses along GBs has already been observed in many other cases, e.g. thorium in tungsten, molybdenum in tungsten, copper in aluminium and copper in nickel. ${ }^{11}$ However the diffusion properties of Te in Ni grain boundaries remains unexplored. The implications of these properties on the performance of MSR experiments motivate such an investigation.

In this work, the diffusion and segregation behaviors of $\mathrm{Te}$ atom along different GB orientations are investigated with the effects of strain included. These results extend our understanding of the physical properties of Te in nickel based alloys and will give a theoretical guide to the experimental work. Then the high corrosion resistant nickel alloys may be manufactured by controlling the concentrations and proportions of different 
types of GBs. Our results on Te segregation and diffusion variation with respect to the GB orientation emphasize the GB misorientation dependence phenomena existed in crystals. ${ }^{12}$

\section{Computational methods}

All calculations in this work were performed with the VASP (Vienna $A b$ initio Simulation Package) $\operatorname{code}^{13}$ with projectoraugmented plane wave (PAW) potentials ${ }^{\mathbf{1 4}}$ and Perdew-BurkeErnzerhof (PBE) exchange-correlation functional. ${ }^{15}$ The wave functions were expanded in a plane-wave basis set with an energy cutoff of $400 \mathrm{eV}$. Spin polarized calculation was included for Ni contained systems to correctly account for the magnetic properties. Monkhorst-Pack scheme ${ }^{16}$ was used to sample the Brillouin zone (BZ). Bulk calculations were carried out using a 3 $\times 3 \times 3$ supercell of the conventional face-centered cubic (fcc) cells with 108 nickel atoms. Supercells for $\Sigma=3$ (111), $\Sigma=5$ (021), $\Sigma=9$ (221) and $\Sigma=11(11 \overline{3})$ GBs contain 192, 120, 160 and 176 atoms, respectively. And the supercells for the investigated surfaces took the same geometry parameters with their corresponding GBs and contained 96, 48, 80 and 88 atoms, respectively. A $3 \times 3 \times 3 k$-point mesh was employed for the bulk calculation. The $k$-point meshes of $1 \times 3 \times 3,2 \times 3 \times 4,1$ $\times 3 \times 3,1 \times 3 \times 3$ were used for $\Sigma=3$ (111), $\Sigma=5$ (021), $\Sigma=9$ (221) and $\Sigma=11$ (11) $)$ GBs and their corresponding surfaces, respectively. After the lattice constants being optimized, the subsequent ionic relaxations were allowed within the cell with the volume and shape of the supercell fixed. The vacancymediated diffusion barriers were calculated by the climbingimage NEB method. ${ }^{17}$ All these calculation setups were checked using larger energy cutoff and denser $k$-mesh; the results of total energy and Hellmann-Feynman forces were convergent within $10^{-6} \mathrm{eV}$ and $0.01 \mathrm{eV} \AA^{-1}$, respectively.

\section{Results and discussions}

\subsection{Te diffusion in bulk nickel and on nickel surfaces}

We first studied the properties of Te atom in the perfect facecentered cubic (fcc) nickel crystal. The optimized lattice parameter of the bulk nickel is $3.515 \AA$, which is consistent with the existing theoretical $(3.518 \AA$ ) and experimental value (3.524 A). ${ }^{3,18}$ The solution energy is the energy required to place a solute atom at a certain lattice site. It is defined as follows:

$$
E_{\mathrm{X}}^{\mathrm{Sol}}=E_{\mathrm{X}}^{\mathrm{bulk}}-\frac{N_{\mathrm{Ni}}}{N_{\mathrm{Ni}}^{0}} E_{\mathrm{Ni}}^{\text {bulk }}-E_{\mathrm{X}}
$$

where $E_{\mathrm{X}}^{\text {bulk }}$ is the energy of the bulk supercell containing the corresponding defect $\mathrm{X} ; E_{\mathrm{Ni}}^{\text {bulk }}$ is the energy of the defect-free Ni bulk supercell; $N_{\mathrm{Ni}}$ and $N_{\mathrm{Ni}}^{0}$ are the numbers of $\mathrm{Ni}$ atoms in the defective Ni bulk supercell and the perfect Ni bulk supercell, respectively; and $E_{\mathrm{X}}$ is the energy per atom of each chemical species in its reference state. Here the reference states refer to the ground-state crystalline phases.

Impurity atoms in a crystal can either occupy the interstitial or substitutional site. The large difference in atomic radii between Te (1.40 A) and Ni (1.24 A) would lead to a large local strain for Te being incorporated interstitially. According to our (a)

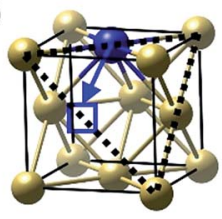

(b)

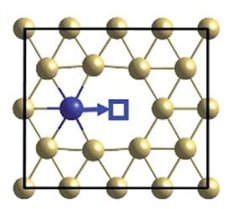

(c)

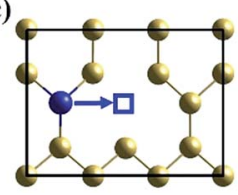

(d)

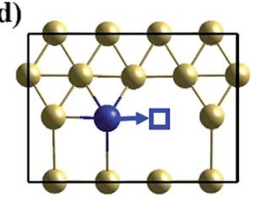

(e)

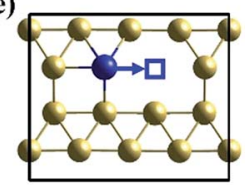

Fig. 1 The diffusion paths of Te atom via vacancy-mediated mechanism for (a) the nickel bulk, (b) the Ni(111), (c) the Ni(021), (d) the Ni(221) and (e) the Ni(113) surfaces. The blue and yellow balls represent $\mathrm{Ni}$ and $\mathrm{Te}$ atoms, respectively. The blue square represents the vacancy and the blue arrow indicates the Te diffusion path.

first-principles calculations, the solution energy of Te atom in the octahedral interstitial site of nickel is $6.29 \mathrm{eV}$ while the value in the tetrahedral interstitial site is even larger, which is $6.93 \mathrm{eV}$. In contrast, the solution energy of Te in the substitutional configuration is only $0.66 \mathrm{eV}$. Since the interstitial tellurium in nickel is significantly high in energy it is unlikely to occur when $\mathrm{Te}$ is incorporated in Ni. Hence, only the substitutional configuration is considered in our further investigation.

As a large solution species, the diffusion of Te atom in nickel is dominated by the vacancy-mediated mechanism, as shown in Fig. 1(a). ${ }^{19-22}$ According to our calculation, the vacancy formation energy in nickel is $1.44 \mathrm{eV}$, which is comparable to that in $\mathrm{Cu}(1.04 \mathrm{eV})$ and $\mathrm{Ir}(1.43 \mathrm{eV})$ obtained by Nazarov et al. ${ }^{23}$ The calculated diffusion barrier of a substitutional Te atom to the nearest nickel vacancy is $0.75 \mathrm{eV}$.

Due to the surface effect, ${ }^{24}$ the properties of Te on different surfaces differ from the bulk properties. The properties of Te on the nickel surfaces were studied by simulating an atomic Te on the top layer of the surfaces. Fig. 1(b-d) depict the investigated

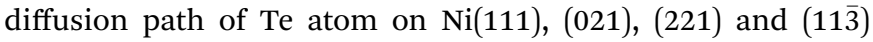
surfaces, respectively. The vacancy formation energy $\left(E_{\mathrm{VNi}}^{\mathrm{F}}\right)$, substitutional Te solution energy $\left(E_{\mathrm{Te}}^{\mathrm{Sol}}\right)$ and the diffusion barrier $\left(B_{\text {Dif }}\right)$ of Te by the vacancy-mediated mechanism on these nickel surfaces are listed in Table 1 . Compared to the $E_{\mathrm{VNi}}^{\mathrm{F}}$ value in the nickel bulk $(1.44 \mathrm{eV})$, the values for the surfaces decrease obviously, indicating that the nickel surfaces should

Table 1 Ni vacancy formation energy ( $E_{\mathrm{VNi}}^{\mathrm{F}}$, in eV), Te substitutional solution energy ( $E_{\mathrm{T}}^{\mathrm{Sol}}$, in $\mathrm{eV}$ ) at the $\mathrm{Ni}$ vacancy site and Te diffusion barrier $\left(B_{\mathrm{Dif}}\right.$ in $\left.\mathrm{eV}\right)$ by the vacancy-mediated mechanism for nickel bulk, $\mathrm{Ni}(111), \mathrm{Ni}(021), \mathrm{Ni}(221)$ and $\mathrm{Ni}(11 \overline{3})$ surfaces

\begin{tabular}{llll}
\hline & $E_{\mathrm{VNi}}^{\mathrm{F}}$ & $E_{\mathrm{Te}}^{\mathrm{Sol}}$ & $B_{\text {Dif }}$ \\
\hline Bulk & 1.44 & 0.66 & 0.75 \\
$(111)$ surface & 1.27 & -1.50 & 0.71 \\
$(021)$ surface & 0.54 & -1.94 & 0.71 \\
$(221)$ surface & 0.63 & -2.02 & 0.89 \\
$(11 \overline{3})$ surface & 0.62 & -1.94 & 0.50
\end{tabular}


have larger vacancy concentrations than the bulk. The $\mathrm{Ni}(021)$ surface has the lowest value of $0.54 \mathrm{eV}$, suggesting the vacancies are easily distributed on this surface. Similarly, the solution energy and the diffusion barrier of Te on surfaces are also decreased. The Ni(221) surface has the lowest $E_{\mathrm{Te}}^{\mathrm{Sol}}$ of $-2.02 \mathrm{eV}$. The dramatically lowered $E_{\mathrm{Te}}^{\text {Sol }}$ suggest that Te atom tends to substitute the $\mathrm{Ni}$ atom on the surfaces. The $\mathrm{Ni}(11 \overline{3})$ surface has the lowest diffusion barrier of Te with the value of only $0.50 \mathrm{eV}$, suggesting a faster diffusion velocity of Te along this type of GB.

\subsection{Te diffusion along the GBs}

3.2.1 Structures and stabilities of the nickel GBs. GBs have a significant effect on the strength and solute diffusion of the $\mathrm{Ni}$
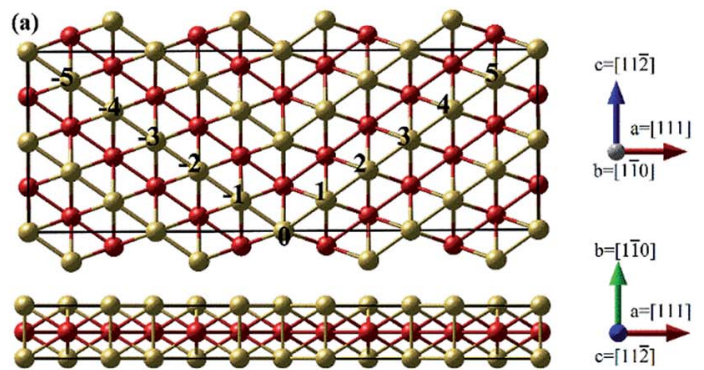

(b)
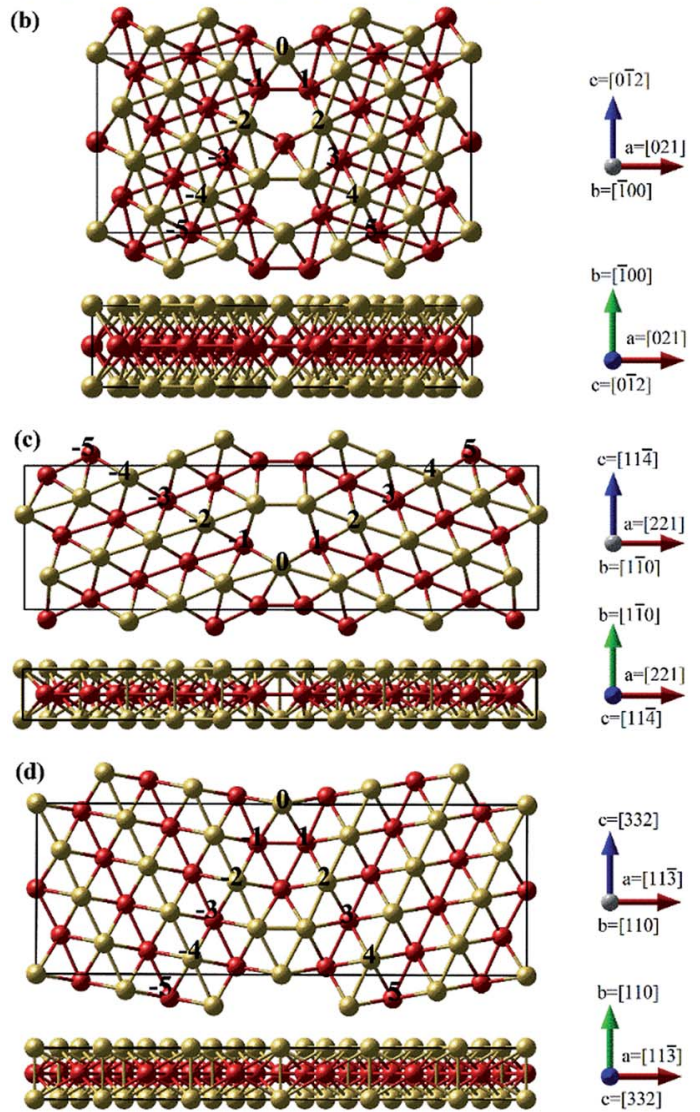

Fig. 2 Side and top views of (a) $\Sigma=3$ (111), (b) $\Sigma=5$ (021), (c) $\Sigma=9$ (221) and (d) $\Sigma=11$ (113) GBs, respectively. The atomic sites are labeled by numbers counted from the GB plane. For clarity, we use the yellow and red balls to represent atoms in adjacent layers (the yellow atoms are in the paper plane, while the red atoms beneath the paper plane). alloy. According to Sangid et al.,$^{25} \Sigma=3$ (111) GB and $\Sigma=11$ $(11 \overline{3}) \mathrm{GB}$ have relatively small grain boundary energies; while the values for $\Sigma=9$ (221) GB and $\Sigma=5$ (021) GB are very high. However, since $\Sigma=9$ (221) GB and $\Sigma=5$ (021) GB can be easily modelled with very few atoms, they are the most widely investigated GBs by theoretical works. ${ }^{3,9,26-31}$ Shen et al. ${ }^{32}$ explored the hydrogen segregation to the $\Sigma=9$ (221) STGB in aluminium. Alexandrov et $a l^{30}$ investigated a wide series of alloying elements diffusing along the $\Sigma=9$ (221) STGB in Ni alloys by $a b$ initio modelling method. Lezzar et al. ${ }^{33}$ analyzed the segregation driving forces for $\mathrm{Ni}(\mathrm{Ag})$ and $\mathrm{Ag}(\mathrm{Ni})$ in the $\Sigma=11(11 \overline{3}) \mathrm{GB}$ using Finnis-Sinclair like potentials. Following these previous studies on the diffusion behavior of many other species at fcc-metal GB, we investigated the Te diffusion at nickel GB by using the same atomic models of Alexandrov et al. ${ }^{30}$ in this work.

As we know, atoms at the GB interface have a different arrangement compared to that in the nickel crystal interior. Fig. 2 shows the structures of $\Sigma=3$ (111), $\Sigma=5$ (021), $\Sigma=9$ (221) and $\Sigma=11(11 \overline{3})$ GBs. Three STGB axes of the investigated four GBs are also depicted in Fig. 2. To obtain the GB structures, the length of the computational box along the direction parallel to the GB interface (axis $b$ and $c$ ) is fixed, while along the other direction perpendicular to the plane of the GB interface (axis $a$ ) the box size is determined based on the lowest grain boundary energy of GB $(\gamma)$ with all the internal atoms fully relaxed. The energy is plotted as a function of the strain, as shown in Fig. 3. The optimized box sizes perpendicular to the $\Sigma=3$ (111), $\Sigma=5$ (021), $\Sigma=9(221)$ and $\Sigma=11(11 \overline{3})$ GBs interface plane are 24.43 $\AA$, $16.45 \AA, 25.22 \AA$ and $23.70 \AA$, respectively.

The stability of the GBs is usually assessed by their grain boundary energies ${ }^{34}$ defined as:

$$
\gamma=\left(E_{\mathrm{tot}}-N E_{\mathrm{coh}}\right) / 2 A
$$

where $E_{\text {tot }}$ is the optimized total energy of the GB supercell, $N$ is the number of atoms in the GB supercell, $E_{\mathrm{coh}}$ is the nickel bulk

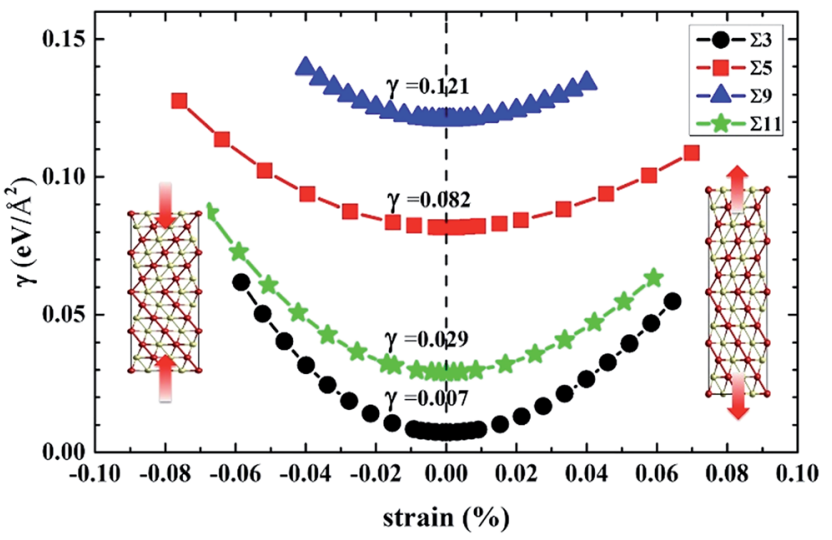

Fig. 3 The optimization of the lattice parameter a (in Å), which is perpendicular to the GB interface plane. The black, red, blue and green lines indicate the grain boundary energy $\left(\gamma\right.$, in eV $\left.\AA^{-2}\right)$ of the GB for $\Sigma=$ 3 (111), $\Sigma=5$ (021), $\Sigma=9$ (221) and $\Sigma=11$ (113) GBs, respectively. The supercells are all relaxed with the lattice parameters parallel to the GB interface plane fixed. The lines joining the calculated quantities are drew as eye guidance. 
cohesive energy and $A$ is the cross-sectional area of the GB supercell. Besides grain boundary energy, excess volume of the GBs can also be used to assess the stability of GBs. Excess volume represents a fundamental key parameter and describes the volume expansion of the GB..$^{35}$ The excess volume is defined as, ${ }^{34}$

$$
\delta V=\left(V_{\text {tot }}-N \Omega_{\text {bulk }}\right) / 2 A
$$

where $V_{\text {tot }}$ is the volume of the GB supercell, $N$ is the number of atoms in the GB supercell, $\Omega_{\text {bulk }}$ is the volume per atom in the bulk crystal and $A$ is the cross-sectional area of the GB supercell.

Our calculated grain boundary energies and excess volume values are listed in Table 2 . The grain boundary energies are ordered in the sequence of $\Sigma=3(111)<\Sigma=11(11 \overline{3})<\Sigma=5$ (021) $<\Sigma=9$ (221). In general the volume expansion of GBs is directly related to the grain boundary energy. ${ }^{36}$ The excess volumes behave approximately linearly with the grain boundary energies, except for the $\Sigma=9$ (221) GB. The large grain boundary energy of $\Sigma=9$ (221) STGB is originated from the fact that $\Sigma=9$ (221) transitional tilt GB is more stable than $\Sigma=9$ (221) STGB. However, we still employed $\Sigma=9$ (221) STGB just like the GB model of Alexandrov et al. ${ }^{30}$ in this work because the diffusion study needs tremendous computing workload for $\Sigma=9$ (221) transitional tilt GB. As shown in Table 2, it is obvious that $\Sigma=3$ (111) GB has the lowest excess volume and grain boundary energy. It is reasonable since the interface plane of $\Sigma=3$ (111) GB is the close-packed (111) surface of nickel in which the atomic arrangement is only slightly distorted compared to that in the bulk interior. In contrast, for the other GBs ( $\Sigma=5(021), \Sigma=9(221)$ and $\Sigma=11$ $(11 \overline{3}))$, the excess volume of these GBs is relatively larger with respect to $\Sigma=3$ (111) and the atomic arrangement is significantly distorted.

3.2.2 The segregation of $\mathrm{Te}$ at $\mathrm{Ni}$ GBs. Te atoms can segregate to the interface plane for the relatively larger activity of GBs. Segregation of Te to nickel GBs leads to GB fracture as a result of embrittlement. The stabilities of Te atom at different atomic sites of GB can be assessed by the value of the segregation energy. The segregation energy is defined as follows:

$$
E_{\mathrm{Seg}}=E_{\mathrm{b}}^{\mathrm{GB}}-E_{\mathrm{b}}^{\mathrm{bulk}}
$$

where $E_{\mathrm{b}}^{\mathrm{GB}}$ is the binding energies for Te at a GB site, and $E_{\mathrm{b}}^{\text {bulk }}$ is the binding energies for Te at the substitutional bulk site. The binding energy of the impurity Te at GB can be obtained as follows: ${ }^{2}$

Table 2 Calculated grain boundary energy $\left(\gamma\right.$, in eV $\left.\AA^{-2}\right)$ and excess volume ( $\delta \mathrm{V}$, in $\AA$ ) for $\Sigma=3$ (111), $\Sigma=5$ (021), $\Sigma=9$ (221) and $\Sigma=11$ (11) GBs of nickel

\begin{tabular}{lllll}
\hline GBs & $\Sigma=3(111)$ & $\Sigma=5(021)$ & $\Sigma=9(221)$ & $\begin{array}{l}\Sigma \\
=11(11 \overline{3})\end{array}$ \\
\hline$\gamma$ & 0.0072 & 0.0818 & 0.1208 & 0.0291 \\
$\delta V$ & 0.0959 & 0.4039 & 0.2652 & 0.2276
\end{tabular}

$$
E_{\mathrm{b}}=E_{\mathrm{GB}, \mathrm{Te}}^{\mathrm{tot}}-E_{\mathrm{Te}}^{\mathrm{bulk}}-E_{\mathrm{GB}}^{\mathrm{tot}}+E_{\mathrm{Ni}}^{\mathrm{bulk}}
$$

where $E_{\mathrm{GB}, \mathrm{Te}}^{\mathrm{tot}}$ refers to the calculated total energy of the $\mathrm{GB}$ supercell including one substitutional Te atom; $E_{\mathrm{Te}}^{\text {bulk }}$ and $E_{\mathrm{Ni}}^{\text {bulk }}$ are the energies of Te atom and $\mathrm{Ni}$ atom in their groundstate crystalline phases, respectively; $E_{\mathrm{GB}}^{\text {tot }}$ is the total energy of the clean GB supercell.

The binding energy $\left(E_{\mathrm{b}}\right)$ and segregation energy $\left(E_{\mathrm{Seg}}\right)$ of Te at different GB sites are calculated for $\Sigma=3(111), \Sigma=5$ (021) and $\Sigma=11(11 \overline{3})$ GBs, as shown in Fig. 4. However, the related results of $\Sigma=9$ (221) GB cannot be obtained, due to the instability of $\Sigma=9$ (221) STGB after Te doping. This instability is owed to the broken symmetry induced by the impurity doping, which is consistent with the previous studies. ${ }^{32,37}$ Although a symmetric $\Sigma=9$ (221) GB is a good model to study the diffusion properties of impurities, ${ }^{30}$ it is not suitable to study the segregation behavior. For $\Sigma=3$ (111), $\Sigma=5$ (021) and $\Sigma=11$ (11̄) GBs, the most active segregation sites are all at the GB interface plane. The segregation energies increase from the interface plane to the bulk area of the GB for all three GBs. For $\Sigma=3$ (111) and $\Sigma=$ $11(11 \overline{3})$ GBs, the relative large segregation energy $(-0.35 \mathrm{eV}$ and $-0.54 \mathrm{eV}$ ) for Te at the interface layer indicates weak segregation. For $\Sigma=5$ (021) GB, the segregation energy is under $-1 \mathrm{eV}$ at the interface. The gradient of its segregation energy is also very large, which indicates a strong tendency for Te segregating to the interface. Therefore, the segregation of Te is most favorable at $\Sigma=5(021) \mathrm{GB}$, while it is the weakest at $\Sigma=3$ (111) GB.
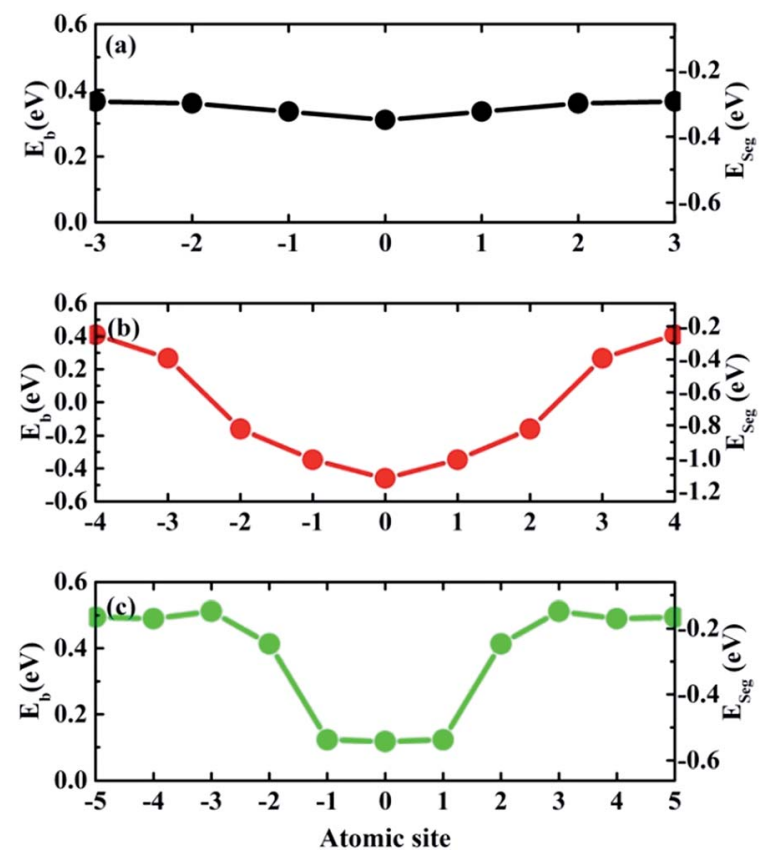

Fig. 4 Calculated binding energy $\left(E_{\mathrm{b}}\right)$ and segregation energy $\left(E_{\text {Seg }}\right)$ for one Te atom at each atomic site (the numbers of atomic sites having been labelled in Fig. 2) in the investigated supercells for (a) $\Sigma=3$ (111), (b) $\Sigma=5$ (021) and (c) $\Sigma=11$ (113) GBs. The lines joining the calculated quantities are drew as eye guidance. 
3.2.3 The embrittling energy of Te at Ni GBs. When a $\mathrm{Ni}$ atom at the interface plane is substituted by a Te atom, the GB structure of the interface region will change. The atomic structures of the clean/Te-doped GBs are depicted in Fig. 5. It is obvious that the structure changes caused by the Te-doping are different for these four types of GBs. The Te atom-induced bond length variation for $\Sigma=9$ (221) GB is the largest, while the variations are small for $\Sigma=5$ (021) and $\Sigma=11$ (11) $\overline{3}$ ) GBs. However, the structural changes of these different GBs have a similar tendency. The bonds perpendicular to the GBs are all elongated greatly. In contrast, the bonds parallel to the GBs are mostly compressed. The expansion induced by the dopant Te across the GB plane could reduce the GB cohesion. Then the Tedoped GBs should fracture more easily than a clean GB, which causes the GB embrittlement.

The embrittlement caused by impurities can also be evaluated by embrittling energy. ${ }^{38}$ According to the Rice-Wang model, the embrittling energy $\left(\Delta E_{\mathrm{E}}\right)$ can be expressed as the difference between the binding energies of Te atoms at the GB $\left(\Delta E_{\mathrm{GB}}\right)$ and the free surface $\left(\Delta E_{\mathrm{FS}}\right)$ :

$$
\Delta E_{\mathrm{E}}=\Delta E_{\mathrm{GB}}-\Delta E_{\mathrm{FS}}
$$

where

$$
\Delta E_{\mathrm{GB}}=E_{\mathrm{I} / \mathrm{GB}}-\frac{N_{\mathrm{Ni}}}{N_{\mathrm{Ni}}^{0}} E_{\mathrm{GB}}-E_{\mathrm{I}}
$$

(a)
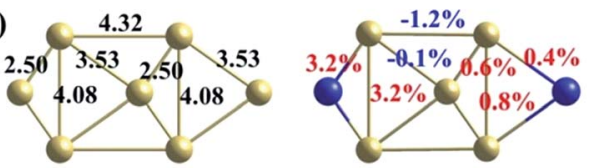

(b)
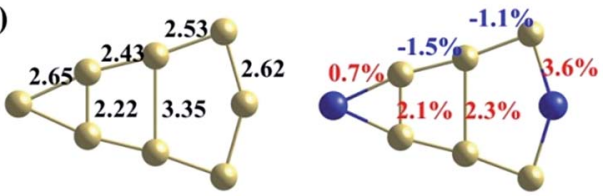

(c)
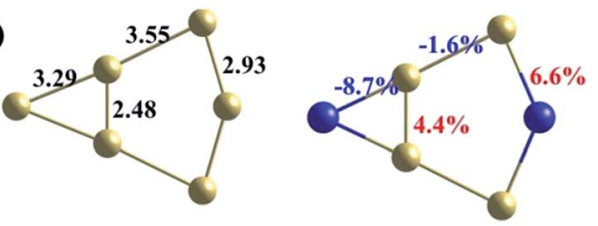

(d)
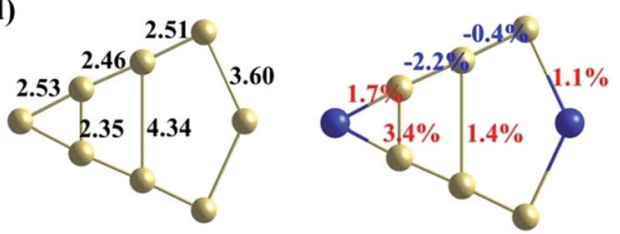

Fig. 5 The atomic structures of the clean GB (left) and the Te-doped GB (right) for (a) $\Sigma=3$ (111), (b) $\Sigma=5$ (021), (c) $\Sigma=9$ (221) and (d) $\Sigma=11$ (113) GBs, respectively. The blue and yellow balls represent $\mathrm{Te}$ and $\mathrm{Ni}$ atom, respectively. On the left panels, the bond lengths (in $\AA$ ) are shown for the clean GBs. On the right panels, the increased/decreased percentage of the corresponding bond length (in \%) are shown for the Te doped GBs. The positive and negative values indicate the bonds are elongated and squashed, respectively. and

$$
\Delta E_{\mathrm{FS}}=E_{\mathrm{I} / \mathrm{FS}}-\frac{N_{\mathrm{Ni}}}{N_{\mathrm{Ni}}^{0}} E_{\mathrm{FS}}-E_{\mathrm{I}}
$$

where $E_{\mathrm{I} / \mathrm{GB}}$ and $E_{\mathrm{I} / \mathrm{FS}}$ are the calculated GB and surface energy with substituted atom, $E_{\mathrm{GB}}$ and $E_{\mathrm{FS}}$ are relaxed clean $\mathrm{GB}$ and surface energy, $N_{\mathrm{Ni}}$ and $N_{\mathrm{Ni}}^{0}$ are the numbers of $\mathrm{Ni}$ atoms in the substituted and clean systems and $E_{\mathrm{I}}$ is the energy of the substituted atom in its reference state.

Based on our calculation, the embrittling energies for $\Sigma=3$ (111), $\Sigma=5$ (021), $\Sigma=9$ (221) and $\Sigma=11$ (11) $)$ GBs are $2.66 \mathrm{eV}$, $1.63 \mathrm{eV}, 1.74 \mathrm{eV}$ and $2.13 \mathrm{eV}$, respectively. In terms of the definition of $\Delta E_{\mathrm{E}}$, a negative value of $\Delta E_{\mathrm{E}}$ means enhancement of the GB cohesion, and a positive value corresponds to GB embrittlement. Therefore, the introduced Te atom would cause embrittlement for all these four GBs in nickel alloy.

3.2.4 The diffusion behavior of Te at Ni GBs. Since Te atoms prefer to segregate into the interfacial sites, we next focus on the diffusion behavior at the interface. Experimental works about Te embrittlement have verified that Te penetrated the nickel GBs along the high diffusivity paths. ${ }^{4,39}$ Fig. 6 shows the investigated diffusion pathway along the four GBs. Among these GBs, the diffusion barrier along the $\Sigma=11$ (11) $)$ GB is the lowest with the value of $0.40 \mathrm{eV} . \Sigma=9$ (221) GB has a relatively small diffusion barrier of $0.66 \mathrm{eV}$. In contrast, the diffusion

(a)

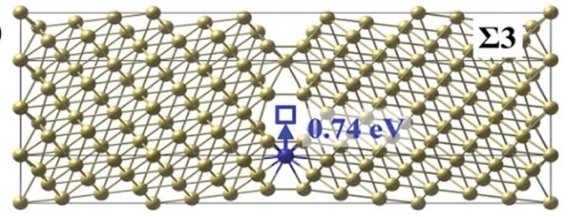

(b)

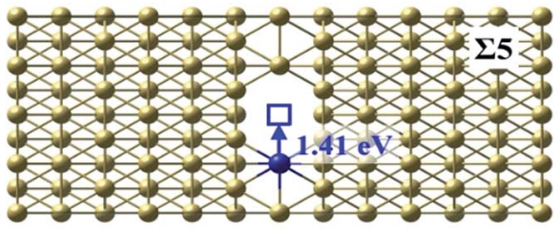

(c)

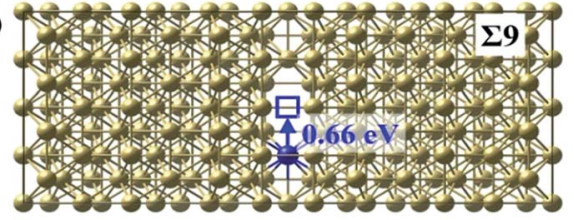

(d)

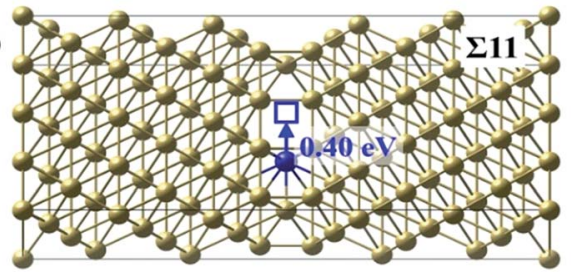

Fig. 6 Top views of $\Sigma=3$ (111), $\Sigma=5$ (021), $\Sigma=9$ (221) and $\Sigma=11$ (113) GBs used to investigate the Te diffusion along the GB via vacancymediated mechanism. The $\mathrm{Ni}, \mathrm{Te}$ atoms and vacancies at GBs are presented by yellow, blue balls and blue squares, respectively. The corresponding diffusion barriers are also indicated. 
barrier of Te along $\Sigma=3$ (111) GB is $0.74 \mathrm{eV}$, which is close to the value in the bulk. This is consistent with our results that the excess volume for $\Sigma=3$ (111) GB is the lowest ( $0.10 \AA)$, indicating its atomic structure is close to the bulk. Surprisingly, $\Sigma=$ 5 (021) GB, which has the highest excess volume, has a very large diffusion barrier of $1.41 \mathrm{eV}$. According to our above results, the diffusion of Te is most likely to occur along $\Sigma=11$ (11) $\overline{3}$ GB, while the commonly used theoretical model $\Sigma=5$ (021) is not favorable for Te diffusion.

In the state of tension, the increasing strain will cause the GB interface to fracture into different surfaces. The stress relief by the formation of the corresponding surfaces could change the diffusion barrier of Te atom. Therefore, the above results for Te diffusion at different GBs are compared with the ones on their corresponding surfaces. As listed in Table 1, the calculated diffusion barriers for Ni(111), (021), (221) and (11 $\overline{3})$ surfaces are $0.71 \mathrm{eV}, 0.71 \mathrm{eV}, 0.89 \mathrm{eV}$ and $0.50 \mathrm{eV}$, respectively. The diffusion barrier value of $0.71 \mathrm{eV}$ on $\mathrm{Ni}(111)$ surface is similar with that at $\Sigma=3$ (111) GB, with a negligible difference of $0.03 \mathrm{eV}$. The Te atom diffusion barrier value on $\mathrm{Ni}(021)$ surface is smaller than the one at $\Sigma=5(021) \mathrm{GB}$ by $0.7 \mathrm{eV}$. In contrast, the Te atom diffusion barrier values on $\mathrm{Ni}(221)$ and $\mathrm{Ni}(11 \overline{3})$ surfaces are both larger than their counterparts at the GBs by $0.23 \mathrm{eV}$ and $0.10 \mathrm{eV}$, respectively.

3.2.5 The strain effect on the diffusion barrier of Te. The synergistic effect of Te embrittlement and strains could cause serious problem of nickel based materials in MSR. ${ }^{5}$ Internal strain occurs inevitably during the application of the nickel alloys, which can be decomposed into the normal one to the GB interface plane and the parallel one. Conventionally, the Te embrittlement caused cracking owes to the normal strain exerted on the GB interface plane. ${ }^{1}$ In order to clarify this synergistic effect, the relation between the strain exerted on the nickel GBs and the diffusion barriers of Te is studied.

Fig. 7 gives the calculated diffusion barrier of Te atom along four kinds of GBs under different strains. Overall, the results show that the interfacial strain has a similar effect on the Te

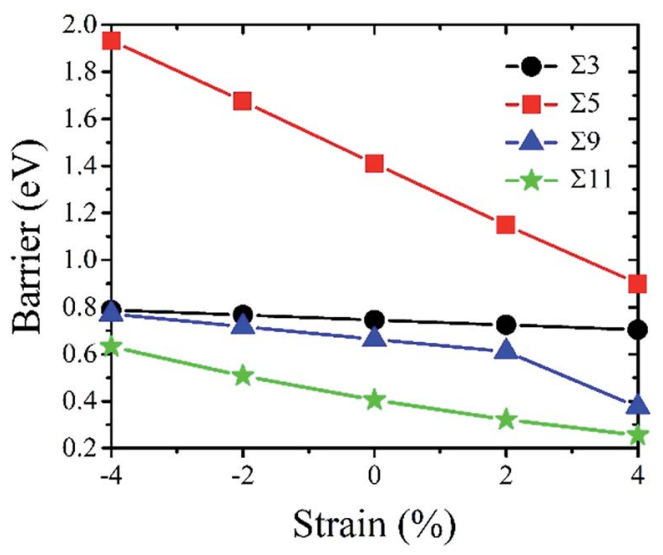

Fig. 7 Diffusion barriers of Te atom for $\Sigma=3$ (111), $\Sigma=5$ (021), $\Sigma=9$ (221) and $\Sigma=11$ (113) GBs with the GBs changing from the compressed state to the strained state. The lines joining the calculated quantities are drew as eye guidance. diffusion behavior at different GBs. The diffusion barriers increase with the GBs under a compressive strain, and decrease under a tensile strain. Therefore, it implies that a tensile strain can increase the diffusivity of Te at nickel GBs. In details, the structures of GBs is also very sensitive to the strain effect. The strain effect for $\Sigma=3$ (111) GB is the smallest and the diffusion barriers' variation is below $0.05 \mathrm{eV}$ for both tension and compression up to $4 \%$. The strain effect for $\Sigma=9$ (221) GB is also relatively small, but the diffusion barrier has a relatively large drop when the tensile strain reach $4 \%$ owing to the fact that $\Sigma=9$ (221) transitional tilt GB is more stable than $\Sigma=9$ (221) STGB. The diffusion barrier for $\Sigma=11$ (11) $\overline{3}) \mathrm{GB}$ is the smallest among the investigated four GBs while the strain effect for this GB is very large. When the tensile strain reaches $4 \%$, the Te diffusion barrier is lowered by $0.15 \mathrm{eV}$. The lowered barrier is only $0.25 \mathrm{eV}$, suggesting that Te diffuses fast along the strained $\Sigma=11(1 \overline{\overline{3}})$ GB. Moreover, it can be found that the $\Sigma=5(021)$ GB exhibits the most remarkable strain effect, and the Te diffusion barrier drops $0.51 \mathrm{eV}$ under a tensile strain of $4 \%$, indicating that $\Sigma=5$ (021) GB is the most sensitive to the tensile strain.
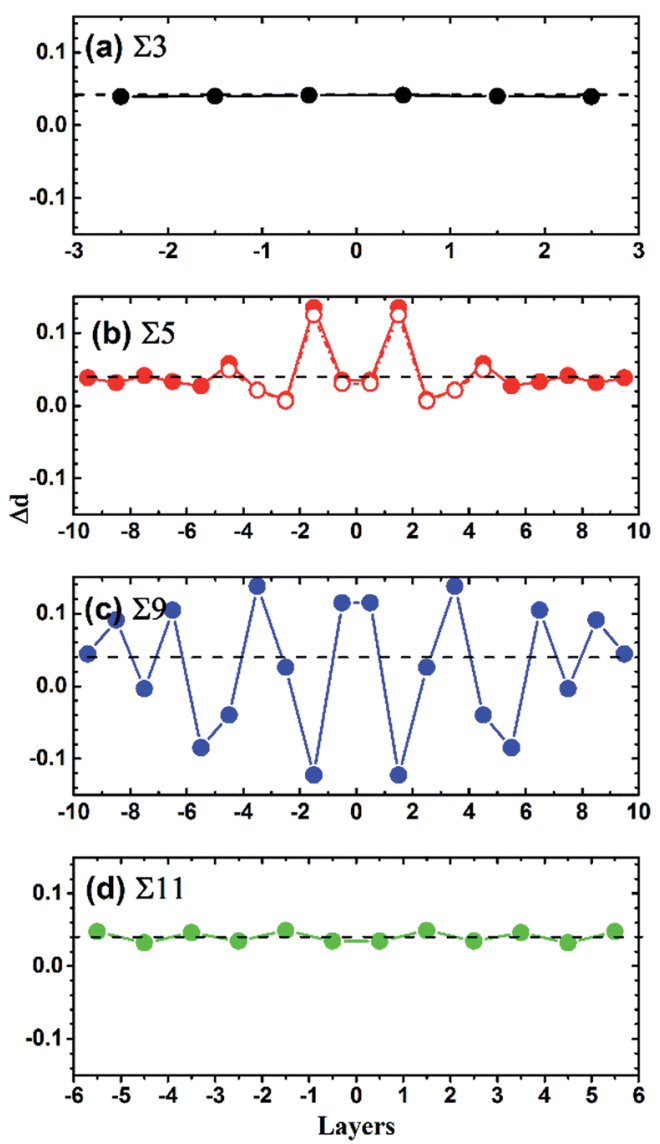

Fig. 8 The variation of interlayer distances with respect to unstrained cases for (a) $\Sigma=3$ (111), (b) $\Sigma=5$ (021), (c) $\Sigma=9$ (221) and (d) $\Sigma=11$ (113) GBs in terms of numbering of layers under $4 \%$ tensile strain. The horizontal line indicates the strain of $4 \%$. The hollow circle in $(b)$ is the data for conventional cell of $\Sigma=5(021) \mathrm{GB}$ and the solid circle in (b) is the data for double slab of $\Sigma=5$ (021) GB. The lines joining the calculated quantities are drew as eye guidance. 
In order to clarify the origin of the strong strain effect on the diffusion barrier of Te at $\Sigma=5$ (021) GB, the variation of interlayer distances $(\Delta d)$ under $4 \%$ tensile strain is analyzed. As shown in Fig. 8, for $\Sigma=3$ (111) and $\Sigma=11$ (11) $\overline{3}$ GBs, their interlayer distances increase uniformly under the tensile strain. In contrast, $\Delta d$ near the interface layer for $\Sigma=9$ (221) GB oscillates strongly. Interestingly, $\Sigma=5$ (021) GB has an obviously different property from the above discussed GBs. As shown in Fig. 8(b), the strain brings about large oscillation of $\Delta d$ in the two layer region adjacent to the GB interface plane and $\Delta d$ is up to $13 \%$. When goes beyond the two layers, the oscillation of $\Delta d$ becomes very small. Therefore, $\Sigma=5$ (021) GB has very large structural variation while being stretched, which explains the strong strain effect on the diffusion barrier of Te. One may argue the $\Sigma=5$ (021) GB has the fewest atoms in our simulations, which may make differences when under a strain. In order to ensure the slab is thick enough to repeat the properties of the $\Sigma=5$ (021) GB, a similar calculation is carried out with the number of layers doubled. As shown in Fig. 8(b), the values obtained by the model with the doubled thickness are consistent with the origin one, indicating the model with 120 atoms is sufficient for $\Sigma=5$ (021) GB.

\section{Conclusions}

In summary, we have systematically studied the properties of Te at nickel STGBs and demonstrated that they are very sensitive to the GB types.

(1) Te is energetically favourable at the substitutional site of nickel for its large atomic volume. Compared to the energy in the nickel bulk, the values of vacancy formation energy, Te substitutional solution energy, and Te diffusion barrier are all decreased obviously on the surfaces, indicating that Te is more easily to diffuse on nickel surfaces by the vacancy-mediated mechanism.

(2) The stability of the GBs is studied by their grain boundary energies. The calculated grain boundary energies are ordered by the following sequences: $\Sigma=3(111)<\Sigma=11$ $(11 \overline{3})<\Sigma=5(021)<\Sigma=9$ (221). And the calculated excess volumes behave approximately linearly to the grain boundary energies, except for the $\Sigma=9$ (221) GB, which originates from the fact that $\Sigma=9$ (221) transitional tilt GB is more stable than $\Sigma=9$ (221) STGB.

(3) The calculated segregation energies indicate that Te has a strong tendency to segregate to the interface layer of the GB. The calculated embrittling energies for nickel GBs are all positive, verifying the embrittling effect of Te atom. The diffusion barrier values are very sensitive to the GB types, and increase in sequence: $\Sigma=11$ (11) $\overline{3}), \Sigma=9(221), \Sigma=3$ (111) and $\Sigma=5$ (021) GBs.

(4) The exploration of the applied strain influence on the diffusion barriers is conducted and the diffusion barriers show obvious variations. Our results imply that a tensile strain can greatly increase the diffusivity of Te at nickel GBs, especially for $\Sigma=5$ (021). This is due to the large structural change near the interface layer caused by strain, which explains the strong strain effect on the diffusion barrier of Te at $\Sigma=5$ (021) GB.

\section{Acknowledgements}

This work was supported by the Program of International S\&T Cooperation (Grant No. 2014DFG60230), National Natural Science Foundation of China (No. 11605273, 91326105, U1404111, 11504089, 21501189, 21676291), the Shanghai Municipal Science and Technology Commission (16ZR1443100), the Strategic Priority Research Program of the Chinese Academy of Sciences (XDA02040104). We also thank the Supercomputing Center of Chinese Academy of Sciences (SCCAS) and the Shanghai Supercomputing Center for computer resources.

\section{Notes and references}

1 W. Liu, H. Han, C. Ren, X. He, Y. Jia, S. Wang, W. Zhang, Z. Li, X. Zhou, Y. Zou, P. Huai and H. Xu, Comput. Mater. Sci., 2014, 88, 22-27.

2 M. Yamaguchi, M. Shiga and H. Kaburaki, Science, 2005, 307, 393-397.

3 M. Všianská and M. Šob, Prog. Mater. Sci., 2011, 56, 817-840. 4 L. Lu, Y. Jia, X.-X. Ye, M. Luo, F. Song, Y. Huang, X. Zhou, Z. Li and Z. Jiang, Corros. Sci., 2016, 108, 169-172.

5 ORNL report No. ORNL/TM-5920, Oak Ridge, Tennessee, US. 6 L. Priester, Grain boundaries and crystalline plasticity, John Wiley \& Sons, 2011.

7 G. S. Rohrer, E. A. Holm, A. D. Rollett, S. M. Foiles, J. Li and D. L. Olmsted, Acta Mater., 2010, 58, 5063-5069.

8 Y. Masatake, S. Motoyuki and K. Hideo, J. Phys.: Condens. Matter, 2004, 16, 3933.

9 D. Di Stefano, M. Mrovec and C. Elsässer, Acta Mater., 2015, 98, 306-312.

10 I. Kaur, Y. Mishin and W. Gust, Fundamentals of grain and interphase boundary diffusion, John Wiley, 1995.

11 R. S. Barnes, Nature, 1950, 166, 1032-1033.

12 G. Gottstein and L. S. Shvindlerman, Grain boundary migration in metals: thermodynamics, kinetics, applications, CRC press, 2009.

13 G. Kresse and J. Furthmüller, Phys. Rev. B: Condens. Matter Mater. Phys., 1996, 54, 11169-11186.

14 P. E. Blöchl, Phys. Rev. B: Condens. Matter Mater. Phys., 1994, 50, 17953-17979.

15 J. P. Perdew, K. Burke and M. Ernzerhof, Phys. Rev. Lett., 1996, 77, 3865-3868.

16 H. J. Monkhorst and J. D. Pack, Phys. Rev. B: Solid State, 1976, 13, 5188-5192.

17 G. Henkelman, B. P. Uberuaga and H. Jónsson, J. Chem. Phys., 2000, 113, 9901-9904.

18 A. Dewaele, M. Torrent, P. Loubeyre and M. Mezouar, Phys. Rev. B: Condens. Matter Mater. Phys., 2008, 78, 104102.

19 H. Z. Fang, S. L. Shang, Y. Wang, Z. K. Liu, D. Alfonso, D. E. Alman, Y. K. Shin, C. Y. Zou, A. C. T. van Duin, Y. K. Lei and G. F. Wang, J. Appl. Phys., 2014, 115, 043501.

20 T. Garnier, V. R. Manga, P. Bellon and D. R. Trinkle, Phys. Rev. B: Condens. Matter Mater. Phys., 2014, 90, 024306.

21 T. Jie, H. Han, W. Darshana, L. Wenguan, Z. Mingwen and H. Ping, J. Phys. D: Appl. Phys., 2014, 47, 215301. 
22 H. Han, D. Wickramaratne, Q. Huang, J. Dai, T. Li, H. Wang, W. Zhang and P. Huai, RSC Adv., 2016, 6, 84262-84268.

23 R. Nazarov, T. Hickel and J. Neugebauer, Phys. Rev. B: Condens. Matter Mater. Phys., 2012, 85, 144118.

24 H. Han and J. G. Che, Appl. Phys. Lett., 2013, 103, 163113.

25 M. D. Sangid, H. Sehitoglu, H. J. Maier and T. Niendorf, Mater. Sci. Eng., A, 2010, 527, 7115-7125.

26 S. Sanyal, U. V. Waghmare, P. R. Subramanian and M. F. X. Gigliotti, Appl. Phys. Lett., 2008, 93, 223113.

27 M. Všianská and M. Šob, Phys. Rev. B: Condens. Matter Mater. Phys., 2011, 84, 014418.

28 E. L. T. Bentria, I. K. Lefkaier and B. Bentria, Mater. Sci. Eng., A, 2013, 577, 197-201.

29 W. Liu, C. Ren, H. Han, J. Tan, Y. Zou, X. Zhou, P. Huai and H. Xu, J. Appl. Phys., 2014, 115, 043706.

30 V. Alexandrov, M. L. Sushko, D. K. Schreiber, S. M. Bruemmer and K. M. Rosso, J. Phys. Chem. Lett., 2015, 6, 1618-1623.
31 D. J. Siegel and J. C. Hamilton, Acta Mater., 2005, 53, 87-96. 32 X. J. Shen, D. Tanguy and D. Connétable, Philos. Mag., 2014, 94, 2247-2261.

33 B. Lezzar, O. Khalfallah, A. Larere, V. Paidar and O. Hardouin Duparc, Acta Mater., 2004, 52, 2809-2818.

34 J. J. Bean and K. P. McKenna, Acta Mater., 2016, 110, 246257.

35 E.-M. Steyskal, B. Oberdorfer, W. Sprengel, M. Zehetbauer, R. Pippan and R. Würschum, Phys. Rev. Lett., 2012, 108, 055504.

36 A. Seeger and G. Schottky, Acta Metall., 1959, 7, 495-503.

37 L. Wan and S. Wang, Phys. Rev. B: Condens. Matter Mater. Phys., 2010, 82, 214112.

38 J. R. Rice and J.-S. Wang, Mater. Sci. Eng., A, 1989, 107, 23-40. 39 H. Cheng, F. Han, Y. Jia, Z. Li and X. Zhou, J. Nucl. Mater., 2015, 461, 122-128. 